\title{
Application of vertex and mass constraints in track-based alignment
}

Chiapolini, N ; Salzmann, C ; Steinkamp, O ; et al

DOI: https://doi.org/10.1016/j.nima.2012.11.192

Posted at the Zurich Open Repository and Archive, University of Zurich ZORA URL: https://doi.org/10.5167/uzh-75674

Journal Article

Accepted Version

Originally published at:

Chiapolini, N; Salzmann, C; Steinkamp, O; et al (2013). Application of vertex and mass constraints in track-based alignment. Nuclear Instruments and Methods in Physics Research Section A: Accelerators, Spectrometers, Detectors and Associated Equipment, 712:48-55.

DOI: https://doi.org/10.1016/j.nima.2012.11.192 


\title{
Application of vertex and mass constraints in track-based alignment
}

\author{
J. Amoraal ${ }^{\mathrm{a}}$, J. Blouw ${ }^{\mathrm{c}}, \mathrm{S}_{\text {S. Blusk }}^{\mathrm{d}}$, S. Borghie,f, M. Cattaneo ${ }^{\mathrm{i}}$,

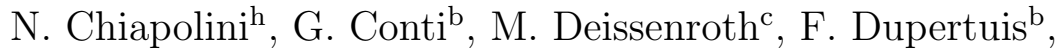 \\ R. van der Eijk ${ }^{\mathrm{a}}$, V. Fave ${ }^{\mathrm{b}}$, M. Gersabeck ${ }^{\mathrm{i}}$, A. Hicheur $^{\mathrm{b}}$, W. Hulsbergen ${ }^{\mathrm{a}, *}$, \\ D. Hutchcroft ${ }^{j}$, A. Kozlinskiy ${ }^{\mathrm{a}}$, R.W. Lambert ${ }^{\mathrm{a}}$, F. Maciuc ${ }^{\mathrm{k}}$, R. Märki ${ }^{\mathrm{b}, *}$, \\ M. Martinellia , M. Merk ${ }^{\mathrm{a}}$, M. Needham ${ }^{\mathrm{l}}$, L. Nicolas ${ }^{\mathrm{b}}$, J. Palacios ${ }^{\mathrm{a}, \mathrm{i}}$, \\ C. Parkes ${ }^{\mathrm{f}}$, A. Pellegrino ${ }^{\mathrm{a}}$, S. Pozzi ${ }^{\mathrm{m}}$, G. Raven ${ }^{\mathrm{a}}$, E. Rodrigues $^{\mathrm{e}, \mathrm{f}}$, \\ C. Salzmann ${ }^{\text {h, M. Schiller }}{ }^{\mathrm{a}}$, O. Schneider ${ }^{\mathrm{b}, *}$, E. Simioni ${ }^{\mathrm{a}}$, O. Steinkamp ${ }^{\mathrm{h}}$, \\ J. van Tilburg ${ }^{\mathrm{c}}$, N. Tuning ${ }^{\mathrm{a}}$, U. Uwer ${ }^{\mathrm{c}}$, S. Vecchi ${ }^{\mathrm{m}}$, S. Viret ${ }^{\mathrm{e}}$ \\ ${ }^{a}$ Nikhef National Institute for Subatomic Physics, Amsterdam, The Netherlands \\ ${ }^{b}$ Ecole Polytechnique Fédérale de Lausanne (EPFL), Lausanne, Switzerland \\ ${ }^{c}$ Physikalisches Institut, Ruprecht-Karls-Universität Heidelberg, Heidelberg, Germany \\ ${ }^{d}$ Syracuse University, Syracuse, $N Y$, United States \\ ${ }^{e}$ School of Physics and Astronomy,University of Glasgow, Glasgow, United Kingdom \\ ${ }^{f}$ School of Physics and Astronomy,University of Manchester, Manchester, United \\ Kingdom \\ ${ }^{g}$ LAL, Université Paris-Sud, CNRS/IN2P3, Orsay, France \\ ${ }^{h}$ Physik-Institut, Universität Zürich, Zürich, Switzerland \\ ${ }^{i}$ European Organization for Nucleaer Research (CERN), Geneva, Switzerland \\ ${ }^{j}$ Oliver Lodge Laboratory, University of Liverpool, Liverpool, United Kingdom \\ ${ }^{k}$ Max-Planck-Institut für Kernphysik (MPIK), Heidelberg, Germany \\ ${ }^{l}$ School of Physics and Astronomy,University of Edinburgh, Edinburgh, United Kingdom \\ ${ }^{m}$ Sezione INFN di Ferrara, Ferrara, Italy
}

\begin{abstract}
The software alignment of planar tracking detectors using samples of charged particle trajectories may lead to global detector distortions that affect vertex and momentum resolution. We present an alignment procedure that constrains such distortions by making use of samples of decay vertices reconstructed from two or more trajectories and putting constraints on their invariant mass. We illustrate the method by using a sample of invariant-mass constrained vertices from $D^{0} \rightarrow K^{-} \pi^{+}$decays to remove a curvature bias in the LHCb spectrometer.
\end{abstract}

Keywords: detector alignment, vertex fit, curvature bias

\footnotetext{
${ }^{*}$ Corresponding author

Email addresses: wouter.hulsbergen@nikhef.nl (W. Hulsbergen), raphael.marki@epfl.ch (R. Märki), olivier.schneider@epfl.ch (O. Schneider)
} 


\section{Introduction}

The calibration of the position and orientation of tracking detectors in high energy physics experiments is called alignment. The input for alignment comes from two sources, namely survey information collected during assembly or after installation, and hit residuals of reconstructed charged particle trajectories (tracks). With track-based algorithms an alignment accuracy can be reached that well exceeds the single-hit resolution.

The track-based alignment algorithms considered here optimize the total fit quality of a sample of tracks, for example the total track fit $\chi^{2}$, with respect to a set of numbers that parametrize the misalignments. The parameters are usually chosen to be the positions and orientations of individual detector elements. We denote the set of alignment parameters with a generic symbol $a$. The condition that the total $\chi^{2}$ be minimal with respect to $a$ can then be written as

$$
\frac{\mathrm{d}}{\mathrm{d} a} \sum_{\text {tracks } i} \chi_{i}^{2}=0,
$$

where the sum runs over all tracks in the calibration sample.

A common problem in the application of track-based alignment algorithms is related to so-called weak modes. These denote alignment degrees of freedom to which the total track $\chi^{2}$ is mostly or completely insensitive. A global translation or rotation of all detector elements with respect to a common point (i.e. a coordinate transformation) is a perfect weak mode. Less trivial weak modes are related to global distortions and depend on the detector geometry. In parallel plane detectors, shearings, such as that depicted in Fig. 1, are also examples of weak modes.

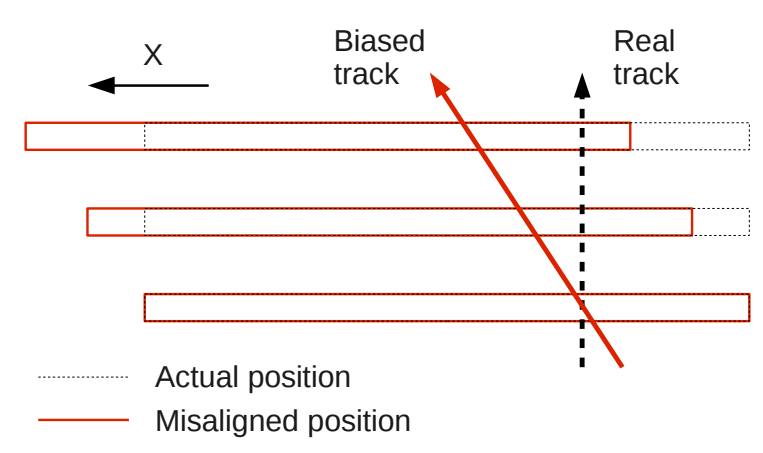

Figure 1: Illustration of a shearing in a planar detector.

One reason weak modes are a concern in alignment is that they lead to poor convergence. An approach to treat weak modes is to impose additional constraints. For example, information on alignment parameters obtained 
from detector survey can be used in the minimization procedure by including additional penalty terms in the $\chi^{2}$.

Weak modes related to global distortions pose a particular concern because they can lead to biases in track parameters that affect the performance of an experiment. A global translation of the entire detection apparatus changes the numerical values of the track parameters without changing the kinematics relevant for a physics analysis, such as an invariant mass or a decay angle. However, global distortions that affect the relative position or direction of tracks will introduce a bias in kinematic observables and degrade the overall detector resolution.

A particularly interesting weak mode related to a global distortion is the so-called curvature bias. This weak mode appears both in cylindrical detectors with a solenoidal magnetic field (in which it is sometimes called sagitta bias or curl) and in forward spectrometers with a dipole magnet, as illustrated in Fig. 2. The momentum of a charged particle is measured via its curvature radius, which requires at least three measured coordinates. In a uniform magnetic field $B$, the inverse of the curvature radius, which we shall call the curvature $\omega$, is related to the momentum component $p_{\perp}$ perpendicular to the field by

$$
\omega=\frac{Q B}{p_{\perp}},
$$

where $Q$ is the charge of the particle. The weak modes illustrated in Fig. 2 introduce a bias in the curvature

$$
\omega \longrightarrow \omega+\delta_{\omega}
$$

that is approximately independent of particle momentum and direction. A constant curvature bias leads to a momentum bias that depends both on momentum and charge. As we shall see later, one signature of a curvature bias is a shift in the reconstructed invariant mass of a two-body decay that is proportional to the momentum difference between the two final-state particles.

The weak mode that leads to a curvature bias only exists in the presence of a the magnetic field. Consequently, a curvature bias can be constrained with field-off data, provided that detectors do not move if the field is turned on. Unfortunately, the latter condition is not often fulfilled. In central detectors a curvature bias can be constrained with samples of reconstructed cosmic ray tracks by exploiting that the 'top' and 'bottom' leg of the track must have the same curvature [1]. In forward detectors, such as the LHCb detector at CERN [2], this technique does not work and alternative methods must be deployed.

We report here on a novel method to constrain the curvature bias by including a $\chi^{2}$ contribution from a mass-constrained vertex fit of a multi-body 

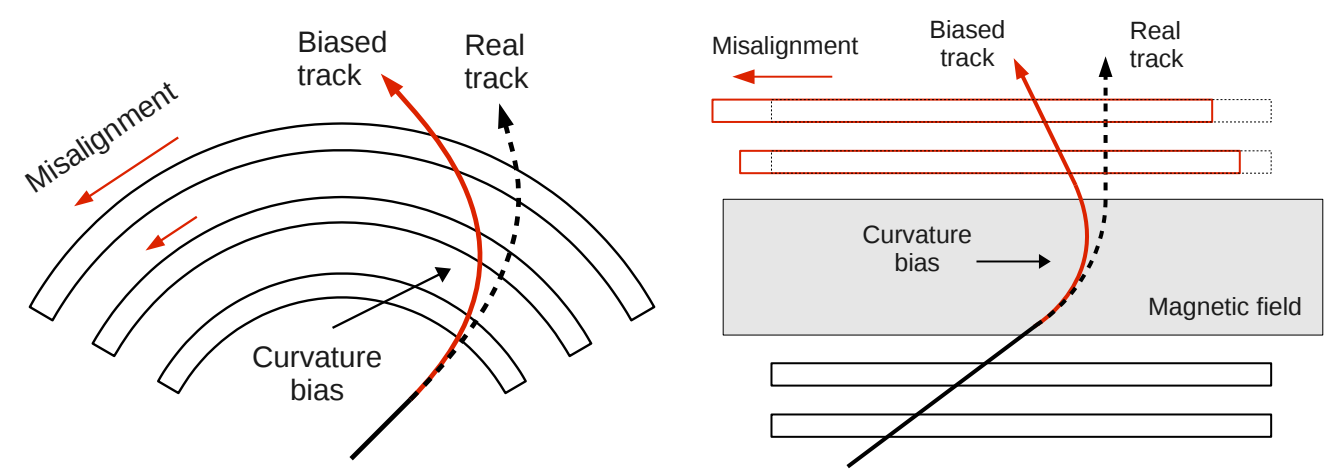

Figure 2: Illustration of a curvature bias in a cylindrical detector geometry (left) and in a forward detector geometry (right).

decay. Implementations of kinematic and vertex constraints for alignment have been presented before $[1,3,4]$. These applications rely on parametrizations of a two-body decay that cannot easily be extended to more than two tracks. Our method does not need a special track parametrization or track fit and can be used with vertices with any number of tracks.

The outline of this paper is as follows. In Section 2 we briefly discuss the track-based alignment procedure, referring the reader to a previous publication for details. In Section 3 we present the implementation of a vertex fit for use in the alignment. In Section 4 we discuss the use of vertex constraints in the alignment of the LHCb detector. As an illustration we compare the performance of alignments obtained with and without mass-contrained $D^{0} \rightarrow K^{-} \pi^{+}$decays. ${ }^{1}$

\section{Alignment with tracks and vertices}

The application of a $\chi^{2}$ minimization procedure for track-based alignment is extensively discussed in the literature (see e.g. Ref. [5] and references therein). For our discussion we use the formalism presented in a previous publication [6], in which we have shown how to use a Kalman-filter track fit in a closed-form alignment procedure. We follow the notation from that paper and from a seminal paper on the application of Kalman filters in high energy physics [7].

We denote the set of alignment parameters by $a$ and the track parameters of track $i$ by $x_{i}$. For a track with $N$ hits, we write the $N$ dimensional vector of hit residuals schematically as

$$
r_{i}\left(x_{i}, a\right)=m_{i}(a)-h_{i}\left(x_{i}\right),
$$

\footnotetext{
${ }^{1}$ Throughout this paper charge-conjugated modes are implied.
} 
where $m_{i}$ is the vector of hit coordinates, each of which is a function of (a subset of) the alignment parameters. The function $h_{i}$ is often called the measurement model. It expresses the expected hit coordinates in terms of the track parameters. ${ }^{2}$ The $\chi^{2}$ of track $i$ can now be written in matrix notation as

$$
\chi_{i}^{2}=r_{i}^{T} V_{i}^{-1} r_{i}
$$

where $V_{i}$ is the $N \times N$ covariance matrix of the measurement coordinates. The latter is usually diagonal. The best-fitting track parameters are obtained by minimizing $\chi_{i}^{2}$ with respect to $x_{i}$ for a given value $a$ of the alignment parameters. Since the measurements depend on $a$, so do the track parameters.

While the track parameters are different for each track, the alignment parameters are common. To obtain the optimal alignment one minimizes the total $\chi^{2}$ of a sample of tracks simultaneously with respect to the track parameters $x_{i}$ and the alignment parameters $a$. By taking into account how the track parameters depend on the alignment parameters, the problem can be reduced to a minimization problem with the dimension of $a$ only $[8,9,10$, $6]$.

Starting from an initial alignment $a_{0}$, the solution for $a=a_{0}+\Delta a$ is obtained by solving the set of linear equations

$$
\left.\frac{\mathrm{d}^{2} \chi^{2}}{\mathrm{~d} a^{2}}\right|_{a_{0}} \Delta a=-\left.\frac{\mathrm{d} \chi^{2}}{\mathrm{~d} a}\right|_{a_{0}}
$$

The first and second derivatives are obtained by summing the contributions from all tracks and can be expressed as $[10,6]$

$$
\frac{\mathrm{d} \chi^{2}}{\mathrm{~d} a}=2 \sum_{\text {tracks } i} \frac{\partial r_{i}^{T}}{\partial a} V_{i}^{-1} r_{i}
$$

and

$$
\frac{\mathrm{d}^{2} \chi^{2}}{\mathrm{~d} a^{2}}=2 \sum_{\text {tracks } i} \frac{\partial r_{i}^{T}}{\partial a} V_{i}^{-1} R_{i} V_{i}^{-1} \frac{\partial r_{i}}{\partial a}
$$

where $R_{i}$ is the covariance matrix of the residuals after the track fit. The latter is expressed as

$$
R_{i}=V_{i}-H_{i} C_{i} H_{i}^{T}
$$

where $H_{i}$ is the derivative of $r_{i}$ with respect to the track parameters of track $i$ and $C_{i}$ is the covariance matrix for the track parameters. To obtain the expression in Eq. 7 one exploits the fact that the $\chi^{2}$ contribution for each

\footnotetext{
${ }^{2}$ Note that one can choose where to put the alignment parameters $a$ in Eq. 4 . The residual can also be written as $r_{i}=m_{i}-h_{i}\left(x_{i}, a\right)[6]$. Only the residual and the derivatives of the residual to track and alignment parameters enter the formalism.
} 
track has been minimized with respect to the track parameters for the initial alignment $a_{0}[6]$.

One ingredient to Eqs. 7 and 8 are the derivatives of the residuals (or measured hit coordinates) to alignment parameters. ${ }^{3}$ Their computation depends on the implementation of the detector geometry and is outside the scope of this paper. ${ }^{4}$ Another ingredient is the track parameter covariance matrix $C_{i}$. In $\mathrm{LHCb}$ we use a Kalman filter track fit that takes multiple scattering and energy loss into account and follow the approach derived in Ref. [6] to compute $C_{i}$.

In Section 4 of Ref. [6] the formalism above is extended with vertex constraints. The proposed method relies on a vertex fit. The vertex fit computes new track parameters on the assumption that all tracks in the fit originate at a common point. Using the covariance matrix of the tracks the difference between the track parameters before and after the vertex fit can be propagated to the hit residuals $r_{i}$. The vertex fit introduces a correlation between the parameters of different tracks. Therefore, the $\chi^{2}$ contributions from different tracks are no longer independent. This means that the residual vector $r_{i}$ now spans residuals from all tracks included in the vertex and the covariance matrix $C_{i}$ is now the covariance matrix for the parameters of all tracks included in the vertex. The method is valid for any number of tracks in a vertex. The strength and novel aspect of the method is that no special track fit is required. The one ingredient that is missing in the discussion in Ref. [6] is the vertex fit itself. We present the vertex fit in the next section.

The actual alignment procedure now consists of the following steps. First, the tracks are fitted. Next, subsets of tracks that are identified to come from a single vertex are combined with a vertex fit. Using the formalism in Ref. [6] a single residual vector $r_{i}$ and corresponding covariance matrix $R_{i}$ are computed for each multi-track object and added to the derivatives in Eqs. 7 and 8. Selected tracks that are not used in a vertex can also be added to these derivatives. Finally, once all contributions in the sample have been accumulated, new alignment parameters are computed using Eq. 6.

\section{The vertex fit}

A vertex fit combines the trajectories of a set of charged particles with the constraint that the particles originate from a common point. The input to the vertex fit are the reconstructed parameters and covariance matrix for each

\footnotetext{
${ }^{3}$ We denote these as partial derivatives since at this stage one ignores the contribution to the derivative that comes through the track parameters.

${ }^{4}$ Their evaluation in LHCb is similar to that discussed in Section 3 of Ref. [10] for the ATLAS detector.
} 
of the tracks. The output of the vertex fit is a vertex position, a momentum vector for each of the tracks and a corresponding covariance matrix.

For the implementation of the vertex fit we use the Billoir-FrühwirthRegler algorithm [11]. We follow the notation used in Ref. [7], with small modifications to remain consistent with the symbols used for the track parameters in the previous section. For the application in alignment we extend the formalism with a mass constraint.

Tracks are locally parametrized by a $5-\mathrm{D}$ vector, generically denoted by the symbol $x_{i}$ where the label $i$ enumerates the tracks in the vertex. The covariance matrix of the track is denoted by $C_{i}$ and its inverse by $G_{i}$. We denote the $3-\mathrm{D}$ vertex position vector with the symbol $\xi$ and the $3-\mathrm{D}$ vector that parametrizes the momentum vector of outgoing track $i$ with $q_{i}$. The measurement model, $h_{i}\left(\xi, q_{i}\right)$ expresses the parameters of track $i$ in terms of $\xi$ and $q_{i}$. The residual of track $i$ is then defined as

$$
r_{i}=x_{i}-h_{i}\left(\xi, q_{i}\right) .
$$

Note that the symbol $x_{i}$ used for the track parameters is the same symbol that appears in Eq. 4 and beyond. However, the role of the track parameters is different: in the track fit, the track parameters are free parameters determined by the track fit. In the vertex fit, the track parameters and their covariance matrix are input to the fit. In the following we show how the track parameters are changed if a vertex constraint is applied.

The $\chi^{2}$ of the vertex fit is written as

$$
\chi^{2}=\sum_{i} r_{i}^{T} G_{i} r_{i}
$$

where the sum runs over all tracks in the vertex. The solution to the vertex fit is the set of parameters $\left(\xi, q_{1}, \ldots, q_{N}\right)$ that minimizes this $\chi^{2}$.

As in Ref. $[7,11]$ we linearize the measurement model around the current estimate $\left(\xi_{0}, q_{i, 0}\right)$,

$$
h_{i}\left(\xi, q_{i}\right)=h_{i}\left(\xi_{0}, q_{i, 0}\right)+A_{i}\left(\xi-\xi_{0}\right)+B_{i}\left(q_{i}-q_{i, 0}\right) .
$$

The measurement model and its derivatives $A_{i}$ and $B_{i}$ follow from the parametrization chosen for tracks and vertices. In Appendix A we shall discuss a definition suitable for a forward detector as LHCb. The total derivatives of the $\chi^{2}$ with respect to the vertex position can now be written as [11]

$$
\frac{\mathrm{d} \chi^{2}}{\mathrm{~d} \xi}=-2 \sum_{i} A_{i}^{T} G_{i}^{B} r_{i} \quad \text { and } \quad \frac{\mathrm{d}^{2} \chi^{2}}{\mathrm{~d} \xi^{2}}=2 \sum_{i} A_{i}^{T} G_{i}^{B} A_{i},
$$

where we introduced

$$
G_{i}^{B} \equiv G_{i}-G_{i} B_{i} W_{i} B_{i}^{T} G_{i}
$$


with

$$
W_{i} \equiv\left(B_{i}^{T} G_{i} B_{i}\right)^{-1} .
$$

The updated vertex parameters are obtained with

$$
\xi=\xi_{0}-\left(\frac{\mathrm{d}^{2} \chi^{2}}{\mathrm{~d} \xi^{2}}\right)^{-1} \frac{\mathrm{d} \chi^{2}}{\mathrm{~d} \xi},
$$

while the covariance matrix for $\xi$ is given by

$$
\operatorname{Cov}(\xi) \equiv C_{\xi}=2\left(\frac{\mathrm{d}^{2} \chi^{2}}{\mathrm{~d} \xi^{2}}\right)^{-1} .
$$

The momentum parameters of the outgoing tracks can be computed with

$$
q_{i}=q_{i, 0}+W_{i} B_{i}^{T} G_{i}\left(x_{i}-h_{i}\left(\xi, q_{i, 0}\right)\right) .
$$

The covariance matrix for two outgoing tracks $i$ and $j$ is given by

$$
\operatorname{Cov}\left(q_{i}, q_{j}\right) \equiv D_{i, j}=\delta_{i j} W_{i}+\left(W_{i} B_{i}^{T} G_{i} A_{i}\right) C_{\xi}\left(A_{j}^{T} G_{j} B_{j} W_{j}\right)
$$

where $\delta_{i j}$ is one if $i=j$ and zero otherwise. The covariance matrix for the vertex position and the momentum of track $i$ is given by

$$
\operatorname{Cov}\left(q_{i}, \xi\right) \equiv E_{i}=-W_{i} B_{i}^{T} G_{i} A_{i} C_{\xi}
$$

The vertex fit can be iterated until a certain convergence criterion is met, for example a sufficiently small change in the vertex $\chi^{2}$ of Eq. 11. Note that the computation of the covariance matrices in Eqs. 19 and 20 is CPU intensive, but not needed for obtaining the $\chi^{2}$ or the position and momentum updates in Eqs. 16 and 18 [11]. Consequently, their computation can be delayed until the fit has converged.

An invariant mass constraint adds a new term to the total vertex $\chi^{2}$ in Eq. 11. In contrast to the $\chi^{2}$ contributions from the individual tracks, the mass $\chi^{2}$ term has non-zero derivatives to all momentum vectors $q_{i}$, instead of just one of them. As a result the optimized formulation of the vertex fit above cannot easily be extended with a mass constraint. Therefore, we have chosen to add the mass constraint after the fit to the vertex position, ignoring small effects due to the non-linearity of the fit.

For each final state particle $i$, we compute a relativistic four-momentum vector $k_{i}$, which is a function of the momentum parameters $q_{i}$ and a mass hypothesis $m_{i}$. We denote the $(4 \times 3)$ matrix for the derivative of $k_{i}$ with respect to $q_{i}$ by

$$
K_{i} \equiv \frac{\mathrm{d} k_{i}}{\mathrm{~d} q_{i}}
$$


The total four-momentum of all tracks assigned to the vertex and its covariance matrix are given by

$$
k_{\mathrm{tot}}=\sum_{i} k_{i}\left(q_{i}, m_{i}\right) \quad \text { and } \quad \operatorname{Cov}\left(k_{\mathrm{tot}}\right)=\sum_{i, j} K_{i} D_{i, j} K_{j}^{T},
$$

where $q_{i}$ and $D_{i, j}$ are the result of the unconstrained fit (Eqs. 18 and 19, respectively). From $k_{\text {tot }}$ we compute the mass and form a residual for the mass constraint,

$$
r_{\mathrm{M}}=m\left(k_{\mathrm{tot}}\right)-m_{0},
$$

where $m_{0}$ is the known mass of the reconstructed decay. Defining the $1 \times 4$ derivative matrix

$$
H_{\mathrm{M}} \equiv \frac{\mathrm{d} m}{\mathrm{~d} k_{\mathrm{tot}}}
$$

the variance of the constraint is given by

$$
R_{\mathrm{M}}=H_{\mathrm{M}} \operatorname{Cov}\left(k_{\mathrm{tot}}\right) H_{\mathrm{M}}^{T} \text {. }
$$

In case the natural width $\Gamma_{0}$ of the decaying particle is not small compared to the invariant mass resolution, such as for the decay $Z^{0} \rightarrow \mu^{+} \mu^{-}$, one can add $\Gamma_{0}^{2}$ to the variance of the constraint.

Using well-known expressions for the Kalman filter [7], the mass-constrained vertex position and track parameters now become

$$
\begin{aligned}
& \hat{\xi}=\xi-\sum_{k} E_{k}^{T} K_{k}^{T} H_{\mathrm{M}}^{T} R_{\mathrm{M}}^{-1} r_{\mathrm{M}}, \\
& \hat{q}_{i}=q_{i}-\sum_{k} D_{i, k} K_{k}^{T} H_{\mathrm{M}}^{T} R_{\mathrm{M}}^{-1} r_{\mathrm{M}} .
\end{aligned}
$$

The updated covariance matrices are given by

$$
\begin{aligned}
\widehat{C}_{\xi} & =C_{\xi}-\sum_{k, l} E_{k}^{T} K_{k}^{T} H_{\mathrm{M}}^{T} R_{\mathrm{M}}^{-1} H_{\mathrm{M}} K_{l} E_{l}, \\
\widehat{D}_{i, j} & =D_{i, j}-\sum_{k, l} D_{i, k} K_{k}^{T} H_{\mathrm{M}}^{T} R_{\mathrm{M}}^{-1} H_{\mathrm{M}} K_{l} D_{l, j}, \\
\widehat{E}_{i} & =E_{i}-\sum_{k, l} D_{i, k} K_{k}^{T} H_{\mathrm{M}}^{T} R_{\mathrm{M}}^{-1} H_{\mathrm{M}} K_{l} E_{l}
\end{aligned}
$$

Finally, for application in the alignment, the vertex-constrained track parameters and their covariance matrices must be computed. These follow from the measurement model as

$$
\tilde{x}_{i}=h\left(\xi, q_{i}\right)
$$




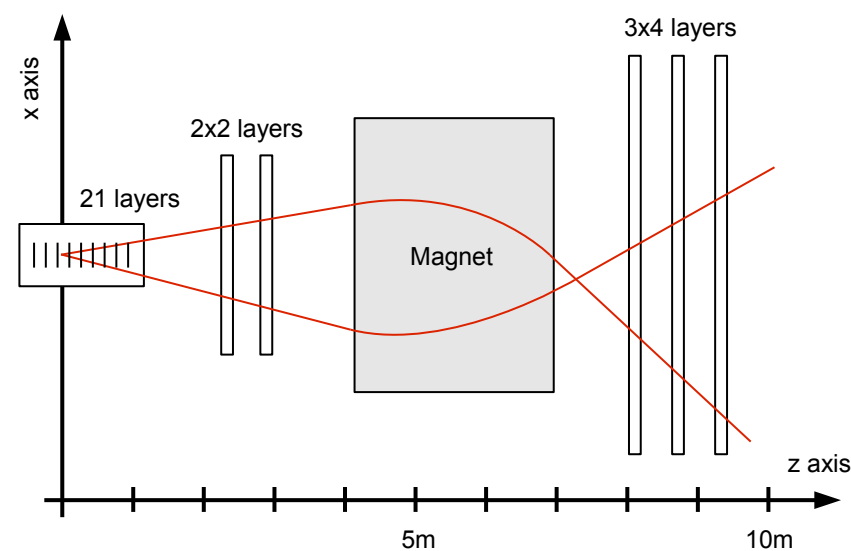

Figure 3: Sketch of the LHCb spectrometer. The proton-proton collision point is located inside the vertex detector on the left $(z \simeq 0)$. The main component of the field of the dipole magnet is parallel to the $y$ axis. The curved lines represent trajectories of a positively and negatively charged particle.

and

$$
\operatorname{Cov}\left(\tilde{x}_{i}, \tilde{x}_{j}\right)=A_{i} C_{\xi} A_{j}^{T}+A_{i} E_{j}^{T} B_{j}^{T}+B_{i} E_{i} A_{j}^{T}+B_{i} D_{i, j} B_{j}^{T} .
$$

These equations provide the input to Eqs. 18, 19 and 21 in Ref. [6]. ${ }^{5}$ In case a mass constraint is used we replace the vertex position $\xi$, the track momentum parameters $q_{i}$ and their corresponding covariance matrices with their massconstrained counterparts in Eqs. 26-30. This concludes the algebra of the vertex fit for use in a track-based alignment algorithm.

\section{Application to the alignment of the $\mathrm{LHCb}$ spectrometer}

The tracking system of the $\mathrm{LHCb}$ detector is an example of a planar detector with a forward geometry. It is schematically depicted in Fig. 3 and discussed in detail in Ref. [2]. Charged particles produced at the interaction point bend in the magnetic field of a dipole magnet with a field integral of about $4 \mathrm{Tm}$. Precision vertexing is provided by a 21-layer silicon strip detector located in the field-free region around the interaction point. Four more layers of silicon strip detectors just in front of the magnet and another 12 layers of silicon strip detectors and straw tube chambers behind the magnet allow for a precise momentum measurement.

The coordinate frame in $\mathrm{LHCb}$ is defined such that the $y$ axis is pointing upwards (parallel to the main component of the dipole field) and the $z$ axis is parallel to the beam line with positive $z$ in the direction of the spectrometer.

\footnotetext{
${ }^{5}$ The symbols $\tilde{x}_{0}^{(i)}, \tilde{C}_{0}^{(i)}$ and $\tilde{C}_{0}^{(i, j)}$ in Ref. [6] translate in our notation as $\tilde{x}_{i}, \operatorname{Cov}\left(\tilde{x}_{i}, \tilde{x}_{i}\right)$ and $\operatorname{Cov}\left(\tilde{x}_{i}, \tilde{x}_{j}\right)$, respectively.
} 
The $x$ axis is chosen such that $(x, y, z)$ is a right-handed system. The origin is located approximately in the center of the vertex detector and roughly corresponds to the average interaction point.

The implementation of a global minimum $\chi^{2}$ algorithm for the alignment of tracking detectors in LHCb has been previously discussed in Ref. [12, 13]. Since then we have extended the algorithm to exploit vertex constraints from primary vertices and from resonances using the techniques outlined above.

\subsection{Primary vertex constraints}

Primary vertices are important for the alignment of the LHCb vertex detector to guarantee an optimal impact parameter and decay time resolution [14]. The silicon modules of the vertex detector are assembled in two detector 'halves' that are positioned on the positive and negative $x$ side of the LHC beam line. The fraction of tracks leaving hits in both halves is small. Furthermore, tracks that cross detector planes both in front and behind the average interaction point (at $z=0$ ) are rare as well. Reconstructed primary vertices allow to link detector planes at positive and negative $x$ and at positive and negative $z$.

We have successfully exploited primary vertices in LHCb alignment using the algorithm described above. Although the algorithm in principle allows to use vertices with an arbitrary number of tracks, a practical problem occurs for high track multiplicity. In LHCb primary vertices often contain tens of reconstructed tracks. Reconstructed tracks can have up to 40 hits. For largemultiplicity primary vertices the computation of the correlations between all hits on all tracks is computationally very demanding. In Ref. [6] it was suggested to compute only the correlations between the hits nearest to the vertex. However, we have found that this can lead to non-positive definite contributions to the second derivative of the $\chi^{2}$ and therefore is not a viable solution.

A working solution has been obtained by limiting the track multiplicity in vertices. For application in alignment we divide a reconstructed primary vertex into separate vertices with at most eight tracks each. Tracks are sorted such that tracks pointing forward, backward, left and right are distributed equally over the different vertices. In our framework the computation time of the correlations in these vertices is small compared to the overall reconstruction and track fitting time. The loss in statistical power due to the splitting of the vertex can be compensated by using more events.

\subsection{Invariant mass constraints}

To constrain weak degrees of freedom in the spectrometer, such as the curvature bias discussed in the introduction, mass-constrained vertices from $D^{0} \rightarrow K^{-} \pi^{+}, J / \psi \rightarrow \mu^{+} \mu^{-}$and $Z^{0} \rightarrow \mu^{+} \mu^{-}$decays are used. The advantage 
of $D^{0} \rightarrow K^{-} \pi^{+}$over other resonances is their large abundance and clean secondary vertex signature in LHCb. This allows to select samples of thousands of events per hour with practically no background.

In order to illustrate the effect on the alignment and on momentum measurements, we show a comparison of two alignment strategies, one obtained using approximately 300k selected high-momentum tracks, and another using in addition the constraint from 80k mass-constraint $D^{0} \rightarrow K^{-} \pi^{+}$vertices in the same sample. For this exercise all alignment parameters for the $\mathrm{LHCb}$ vertex detector were fixed, while all detector elements behind the vertex detector were allowed to move in the $x$ direction and rotate in the $x y$ plane around their center of gravity. To constrain weak modes and ensure convergence, survey information was used by adding for each alignment parameter a penalty term to the total $\chi^{2}$ constraining it to its survey value within the uncertainty assigned to the survey. The alignment process started in both cases from alignment parameters obtained with early data $[12,15]$.

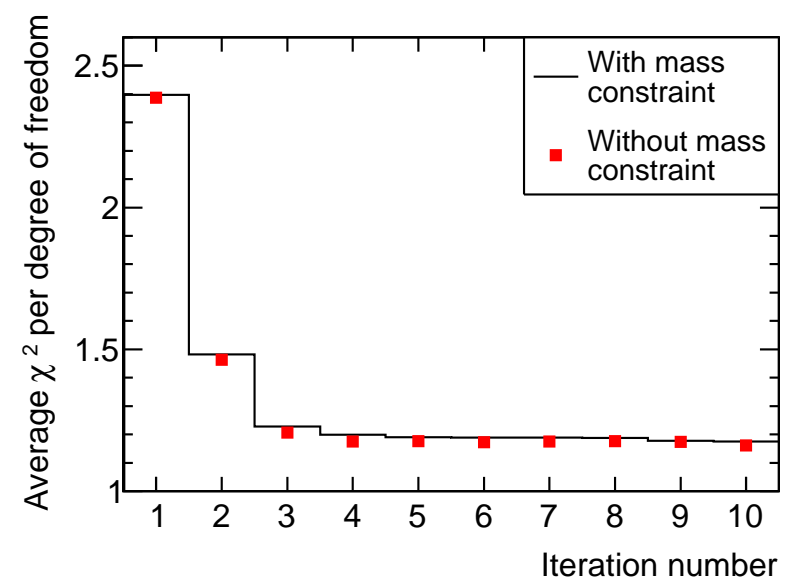

Figure 4: Average $\chi^{2}$ per degree of freedom at each iteration of the alignment procedure for the alignment with (black line) and without (red squares) $D^{0}$ mass constraint.

In both scenarios the minimization converged quickly as illustrated by the average $\chi^{2}$ per degree of freedom versus iteration, shown in Fig. 4. Although the average track $\chi^{2}$ after the alignment is practically identical in the two cases, the performance in terms of invariant mass resolution is very different. Figure 5 shows the invariant mass distribution of $D^{0} \rightarrow K^{-} \pi^{+}$and $J / \psi \rightarrow \mu^{+} \mu^{-}$candidates on independent data sets using the two alignment sets as input. The mass resolution obtained with the alignment that exploits the $D^{0} \rightarrow K^{-} \pi^{+}$mass constraint is considerably better.

To understand this behaviour we consider the effect of a curvature bias on the invariant mass of a decay to two oppositely charged particles with momenta $p_{-}$and $p_{+}$and masses $m_{-}$and $m_{+}$, respectively. Assuming small 

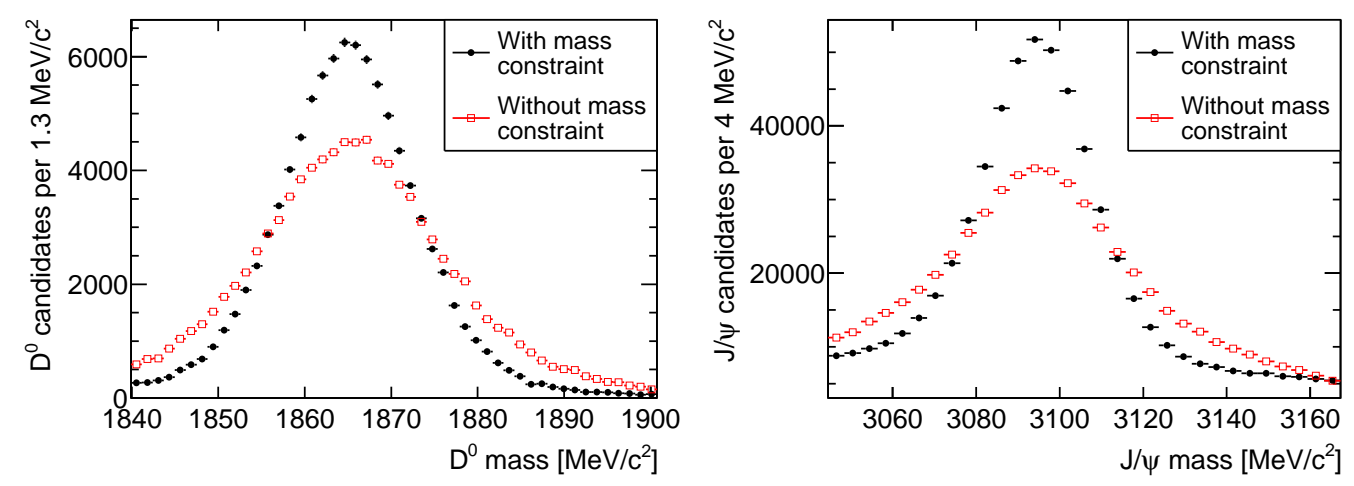

Figure 5: Invariant mass distribution of $D^{0} \rightarrow K^{-} \pi^{+}$(left) and $J / \psi \rightarrow \mu^{+} \mu^{-}$(right) candidates obtained with the alignment using the $D^{0}$ mass constraint (black solid points) and the alignment based only on tracks (red open points).

masses with respect to the momenta, i.e. ignoring terms of order $m_{i}^{2} / p_{i}^{2}$, the invariant mass is given by

$$
m=\sqrt{m_{-}^{2}+m_{+}^{2}+2 p_{-} p_{+}(1-\cos \theta) / c^{2}}
$$

where $\theta$ is the opening angle between the two particles. As a result of a curvature bias $\delta_{\omega}$, the momentum changes as

$$
p_{\perp} \longrightarrow p_{\perp}\left(1-\frac{\delta_{\omega}}{\omega}\right)=p_{\perp}\left(1-\frac{\delta_{\omega} p_{\perp}}{Q B}\right)
$$

where we have ignored higher order terms in $\delta_{\omega} / \omega$. Note that the sign of the bias in the momentum is opposite for the positive and the negative track. In a forward spectrometer the momentum component perpendicular to the field dominates the total momentum. The change in the invariant mass then becomes, to first order in $\delta_{\omega} / \omega$,

$$
m \longrightarrow m\left(1-\left(p_{+}-p_{-}\right) \frac{m^{2}-m_{-}^{2}-m_{+}^{2}}{2 m^{2}} \frac{\delta_{\omega}}{e B}\right)
$$

where $e$ is the positron charge. In other words, one expects a bias in the mass that is approximately proportional to the difference of the momenta of the two final state particles.

This effect is demonstrated in Fig. 6. The figure shows the position of the peak of the invariant mass distribution for $D^{0} \rightarrow K^{-} \pi^{+}$and $J / \psi \rightarrow \mu^{+} \mu^{-}$ decays as a function of the momentum difference of the final state particles in the two scenarios. Indeed, if the mass constraint is not used a clear evidence of a curvature bias is observed. The removal of the curvature bias explains the difference in mass resolutions shown in Fig. 5. 

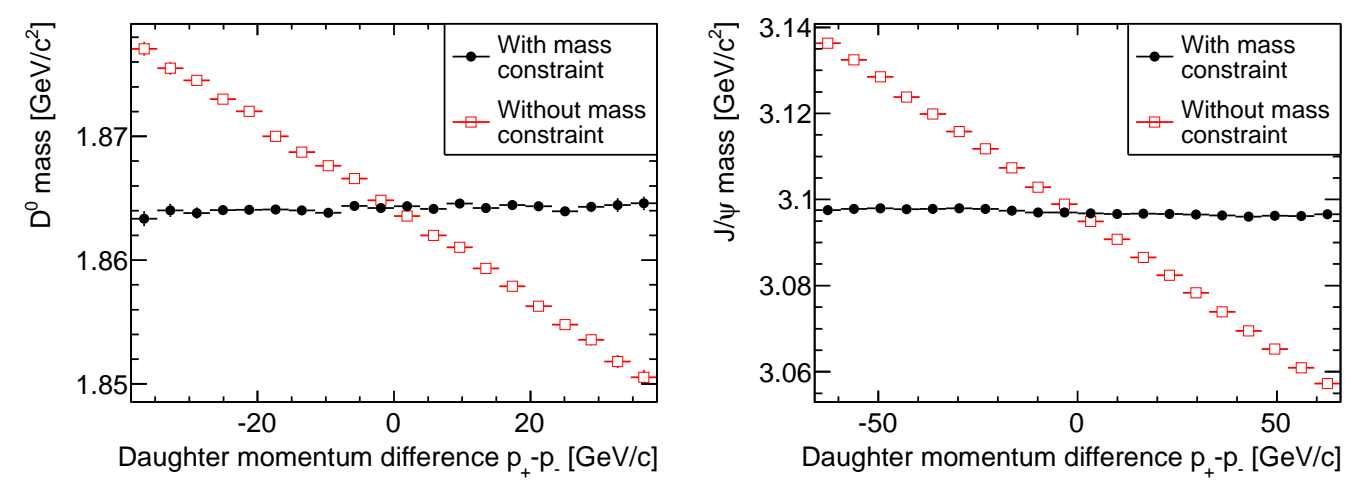

Figure 6: Position of the peak of the invariant mass distribution for $D^{0} \rightarrow K^{-} \pi^{+}$(left) and $J / \psi \rightarrow \mu^{+} \mu^{-}$(right) candidates as a function of the momentum difference of the two daughter tracks evaluated with the alignment using the $D^{0}$ mass constraint and the alignment based only on tracks.

From the slopes of the graphs in Fig. 5 one can estimate the value of $\delta_{\omega} / 2 e B$. We verify that the two slopes are consistent with a single curvature bias. Studies with simulated LHCb data documented in Ref. [16] show that the curvature bias is proportional to the $x$ displacement of the detector planes behind the magnet. The reported constant of proportionality allows us to predict the displacement from the observed slopes in Fig. 5. We compute a value of approximately $1.8 \mathrm{~mm}$ for the $x$ movement of the tracking layers behind the magnet, consistent with the actually observed difference in alignment constants between the scenarios with and without mass constraint.

As explained in the introduction a curvature bias may appear in the scenario without the mass constraint because it corresponds to a weak mode, a common displacement of tracking layers that is not constrained by single particle trajectories. Weak modes are constrained in the LHCb alignment by survey information. The precision of the survey is poor compared to the resolution of individual tracks. However, since tracks carry no information on the weak modes, in the absence of mass constraints the precision of the survey limits the precision to which the curvature bias can be constrained. The $2 \mathrm{~mm}$ difference between the two alignments seems large compared to the typical uncertainty of the survey information for the detector layers behind the magnet, which is about $0.5 \mathrm{~mm}$. However, for the results obtained here the vertex detector was used as a reference. The observed displacement is equivalent to a $0.2 \mathrm{mrad}$ rotation of the vertex detector around its $y$ axis, consistent with the precision of the vertex detector survey.

\section{Conclusion}

Following a recipe outlined in Ref. [6] we have developed a method to extend a track-based minimum $\chi^{2}$ algorithm for detector alignment with 
information from reconstructed vertices. We have presented the algebra of the vertex fit, including a mass constraint. We have demonstrated how such a vertex fit can be exploited to remove the effect of a curvature bias in the alignment procedure. For $\mathrm{LHCb}$, invariant mass resolution is important to isolate rare $B$ decays from the background and to separate decays that are kinematically close, such as decays of $B$ mesons to two light hadrons. Mass constraints are now part of the standard $\mathrm{LHCb}$ alignment procedure and allow the LHCb collaboration to perform world-best measurements in such decays [17].

\section{Acknowledgements}

The authors of this paper are members and ex-members of the LHCb collaboration. For the analysis presented in Section 4 we are indebted to the $\mathrm{LHCb}$ collaboration for the use of the LHCb software environment and $\mathrm{LHCb}$ data. Individual authors acknowledge support from various National Agencies, in particular CERN, CNRS/IN2P3 (France), BMBF, DFG, HGF and MPG (Germany), INFN (Italy), FOM and NWO (The Netherlands), SNSF and SER (Switzerland), STFC (United Kingdom) and NSF (USA).

\section{Appendix A. Track and vertex model for a forward detector}

Here we discuss a parametrization for the vertex fit in a forward detector like LHCb. We define a cartesian coordinate frame as in Section 4. Track trajectories are locally parametrized by a 5 -D 'state' vector $\left(x, y, t_{x}, t_{y}, \kappa\right)$ at a given $z$ coordinate, such that $(x, y, z)$ is a point on the track, $t_{x}$ and $t_{y}$ are the local tangents $\mathrm{d} x / \mathrm{d} z$ and $\mathrm{d} y / \mathrm{d} z$, respectively, and $\kappa=Q /(p c)$ with $Q$ the charge and $p$ the momentum.

In the vertex fit the vertex coordinates $\xi$ are parametrized by a vector $\xi=\left(x_{v}, y_{v}, z_{v}\right)$. The momentum vector of each of the outgoing tracks $i$ is parametrized by a 3 -D vector $q_{i}=\left(t_{x, v, i}, t_{y, v, i}, \kappa_{v, i}\right)$, where the meaning of parameters is the same as in the measured track state. The subscript $v$ indicates that these are now parameters of the fit, not reconstructed track parameters. The motivation for choosing this particular parametrization is that it makes the measurement model nearly linear.

We assume that the reference $z$ coordinate of each of the track states is sufficiently close to the $z$ coordinate of the vertex that the magnetic field can be ignored. The measurement model for track $i$ then becomes

$$
h\left(\xi, q_{i}\right)=\left(\begin{array}{c}
x_{v}+\left(z_{i}-z_{v}\right) t_{x, v, i} \\
y_{v}+\left(z_{i}-z_{v}\right) t_{y, v, i} \\
t_{x, v, i} \\
t_{y, v, i} \\
\kappa_{v, i}
\end{array}\right)
$$


where $z_{i}$ is the position at which the measured state vector of the track is defined. For the corresponding derivative matrices we obtain

$$
A\left(\xi, q_{i}\right)=\left(\begin{array}{ccc}
1 & 0 & -t_{x, v, i} \\
0 & 1 & -t_{y, v, i} \\
0 & 0 & 0 \\
0 & 0 & 0 \\
0 & 0 & 0
\end{array}\right)
$$

and

$$
B\left(\xi, q_{i}\right)=\left(\begin{array}{ccc}
z_{i}-z_{v} & 0 & 0 \\
0 & z_{i}-z_{v} & 0 \\
1 & 0 & 0 \\
0 & 1 & 0 \\
0 & 0 & 1
\end{array}\right)
$$

Note that in the absence of a magnetic field, the magnitude of the momentum is not required to obtain the vertex position. Therefore, the fit can be performed more efficiently by omitting the curvature parameter altogether and working with a 4 -D track model. The updated curvature can then be computed afterward by propagating the change in the remaining four track parameters. This approach requires more algebra, in particular when implementing the mass constraint. Consequently, we have not used it.

For completeness we also present the expressions for the derivative matrices needed for the mass constraint. We drop the subscript $v$ for readability and choose units such that $c=1$. In terms of the fit parameters the fourvector $\left(\vec{k}_{i}, k_{0, i}\right)$ of outgoing track $i$ becomes

$$
k_{i}=\left(\begin{array}{c}
p_{i} t_{x, i} / n_{i} \\
p_{i} t_{y, i} / n_{i} \\
p_{i} / n_{i} \\
\sqrt{m_{i}^{2}+p_{i}^{2}}
\end{array}\right)
$$

where $m_{i}$ is the track candidate mass, $p_{i}=Q_{i} / \kappa_{i}$ and $n_{i}=\sqrt{1+t_{x, i}{ }^{2}+t_{y, i}{ }^{2}}$. The derivative matrix in Eq. 21 is then given by

$$
K_{i}=\left(\begin{array}{ccc}
\left(1+t_{y, i}^{2}\right) p_{i} / n_{i}^{3} & -t_{x, i} t_{y, i} p_{i} / n_{i}^{3} & -Q_{i} t_{x, i} p_{i}^{2} / n_{i} \\
-t_{x, i} t_{y, i} p_{i} / n_{i}^{3} & \left(1+t_{x, i}^{2}\right) p_{i} / n_{i}^{3} & -Q_{i} t_{y, i} p_{i}^{2} / n_{i} \\
-t_{x, i} p_{i} / n_{i}^{3} & -t_{y, i} p_{i} / n_{i}^{3} & -Q_{i} p_{i}^{2} / n_{i} \\
0 & 0 & -Q_{i} p_{i}^{3} / \sqrt{m_{i}^{2}+p_{i}^{2}}
\end{array}\right) .
$$

Finally, taking the vertex invariant mass as the norm of the total four-vector, $m=\sqrt{k_{0, \text { tot }}^{2}-\left|\vec{k}_{\text {tot }}\right|}$, one obtains for the derivative matrix Eq. 24 of the mass constraint

$$
H_{M}=\left(-k_{x, \text { tot }} / m,-k_{y, \text { tot }} / m,-k_{z, \text { tot }} / m, k_{0, \text { tot }} / m\right) .
$$




\section{References}

[1] D. Brown, A. Gritsan, Z. Guo, and D. Roberts, Local alignment of the $B A B A R$ silicon vertex tracking detector, Nucl. Instrum. Meth. A603 (2009) 467, arXiv:0809.3823.

[2] LHCb collaboration, A. A. Alves Jr et al., The LHCb detector at the LHC, JINST 3 (2008) S08005.

[3] G. Flucke, Alignment of the CMS Silicon Tracker, CERN-CMS-CR2011-323, 2011.

[4] E. Widl and R. Frühwirth, Application of the Kalman alignment algorithm to the CMS tracker, J. Phys. Conf. Ser. 219 (2010) 032065.

[5] V. Blobel, Software alignment for tracking detectors, Nucl. Instrum. Meth. A566 (2006) 5.

[6] W. Hulsbergen, The global covariance matrix of tracks fitted with a Kalman filter and an application in detector alignment, Nucl. Instrum. Meth. A600 (2009) 471, arXiv:0810.2241.

[7] R. Frühwirth, Application of Kalman filtering to track and vertex fitting, Nucl. Instrum. Meth. A262 (1987) 444.

[8] V. Blobel and C. Kleinwort, A new method for the high precision alignment of track detectors, arXiv:hep-ex/0208021.

[9] P. Brückman, A. Hicheur, and S. J. Haywood, Global $\chi^{2}$ approach to the alignment of the ATLAS silicon tracking detectors, CERN-ATLINDET-PUB-2005-002, 2005.

[10] A. Bocci and W. Hulsbergen, TRT alignment for SR1 cosmics and beyond, CERN-ATL-COM-INDET-2007-011, 2007.

[11] P. Billoir, R. Frühwirth, and M. Regler, Track element merging strategy and vertex fitting in complex modular detectors, Nucl. Instrum. Meth. A241 (1985) 115.

[12] L. Nicolas et al., Alignment of LHCb tracking stations with tracks fitted with a Kalman filter, CERN-LHCB-2008-066, LPHE-2008-14, 2008.

[13] J. Amoraal, Alignment of the LHCb detector with Kalman filter fitted tracks, J. Phys. Conf. Ser. 219 (2010) 032028.

[14] S. Viret, C. Parkes, and M. Gersabeck, Alignment procedure of the LHCb Vertex Detector, Nucl. Instrum. Meth. A596 (2008) 157, arXiv:0807.5067. 
[15] M. Gersabeck, S. Viret, and C. Parkes, Performance of the LHCb vertex detector alignment algorithm determined with beam test data, Nucl. Instrum. Meth. A596 (2008) 164, arXiv:0807.5069; S. Borghi et al., First spatial alignment of the LHCb VELO and analysis of beam absorber collision data, Nucl. Instrum. Meth. A618 (2010) 108.

[16] E. E. Simioni, New physics from rare beauty. PhD thesis, Vrije Universiteit, Amsterdam, The Netherlands, 2010. CERN-THESIS-2010-031.

[17] LHCb collaboration, R. Aaij et al., First evidence of direct CP violation in charmless two-body decays of $B_{s}$ mesons, Phys. Rev. Lett. 108 (2012) 201601, arXiv:1202.6251; LHCb collaboration, R. Aaij et al., Strong constraints on the rare decays $B_{s} \rightarrow \mu^{+} \mu^{-}$and $B^{0} \rightarrow \mu^{+} \mu^{-}$, Phys. Rev. Lett. 108 (2012) 231801, arXiv:1203.4493. 